\title{
Super-resolution using Interpolation and Wavelet Lifting Scheme
}

\author{
Sapan Naik \\ Department of Computer Engg , \\ Government Engineering College, Sector 28, \\ Gandhinagar, India
}

\author{
Viral Borisagar \\ Asst. Professor \\ Department of Computer Engg , \\ Government Engineering College, Sector 28, \\ Gandhinagar, India
}

\begin{abstract}
Due to the limitation of image capturing technique, we often get low resolution(LR) images. We can construct high resolution(HR) images from one or more LR images and this method is called Super Resolution(SR). In this paper we have proposed one lifting based super resolution algorithm which generate super resolution image from three rotated, noisy and blurred images. We have used HAAR wavelet lifting scheme and ASWDR encoding method. To remove noise and blur from original images we have applied Gaussian filter and Iterative Blind Deconvolution(IBD) algorithm.
\end{abstract}

\section{Keywords}

Resolution, Restoration, Wavelet lifting scheme.

\section{INTRODUCTION}

Images are often down sampled and transmitted at low bit rates resulting in a low resolution(LR) compressed image, due to the factors like processing power limitations and channel capabilities. High resolution(HR) images can be reconstructed from several blurred, noisy and down sampled low resolution images using a computational process know as super resolution(SR) image reconstruction.

In SR techniques, The basic premise for increasing the spatial resolution is the availability of multiple LR images captured from the same scene [9]. In SR, for the same scene we have different images with different looks. That is, LR images are aliased as well as shifted with sub pixel precision. Many different methods are available to do Super Resolution like nonuniform interpolation, frequency domain approach, regularization. [7,8].

A related problem to SR techniques is image restoration, which is a well-established area in image processing applications [10]. Image restoration recover a degraded image, but it does not change the size of image like SR.

In this paper we have used wavelet based lifting because we can achieve high compression ratio as well as improve the quality of the image by adding detail information. Wavelets are good mathematical tool for hierarchically decomposing functions and it allow efficient algorithm as it can be computed in linear time.

This paper is organized as follows. In Section II, We describe Image registration and IBD algorithm; Section III we describe HAAR wavelet lifting scheme and ASWDR Coding Scheme for wavelet coefficient, In Section IV we present our proposed super resolution algorithm ; section $\mathrm{V}$ consists of experiment results and finally in section VI Conclusion.

\section{IMAGE REGISTRATION AND IBD ALGORITHM}

Image registration is performed first in order to align the LR images as accurately as possible for super resolution reconstruction. Using registration we can overlap two or more images of the same scene taken at different times or from different view points or by different sensors. From all these images one image is called the base image which is considered as reference to which the other input images are compared. The purpose of registration is to bring the input image into alignment with the base image by applying a spatial transformation to the input image. Spatial transformation maps locations in an image into a new location in other image. Registration dependent on the properties of the camera like resolution of sensor, the noise of the device and the imperfection of the lens that adds blur. The local two dimensional structure of an image degrades and an exact registration of two low resolution images becomes increasingly difficult as the resolution decreases. High quality registration requires for Super resolution reconstruction. Many different methods exists for estimating the motion or sub pixel shift between the two images. The registration technique considered in our method is a modified phase correlation based on Fast Fourier Transform proposed by Fourier Mellin and DeCastro[12].

We can preprocessed images using Gaussian filter and IBD algorithm. We can apply Gaussian filter to remove noise from the image and same way we can apply IBD algorithm to remove blur from the image.

We often get blurred and degraded images due to relative motion between camera and object. To reconstruct the original image we need deblurring algorithm. One of the popular blind deconvolution algorithms is the iterative blind deconvolution (IBD) algorithm proposed by [11]. The IBD algorithm iteratively estimates the original image as well as the PSF. IBD makes use of spatial domain as well as frequency domain constraints. In spatial domain, positivity constraint is used on both the image as well as PSF. Positivity is used in spatial domain because image pixel intensity values are always positive.

\section{LIFTING SCHEME AND CODING METHOD}

An image can be analyzed by passing it through an analysis filter bank followed by a decimation operation in discrete wavelet transform. Analysis filter bank consists of a low pass and a high pass filter at each decomposition stage. When a 
signal passes through these filters, it is split into two bands. The low pass filter extracts the coarse information of the signal, which corresponds to an averaging operation. The high pass filter extracts the detail information of the signal, which corresponds to a differencing operation. The output of the filtering operations is then decimated by two[3].

A two-dimensional transform can be accomplished by performing two separate one-dimensional transforms. First, the image is filtered along the $\mathrm{x}$-dimension using low pass and high pass analysis filters and decimated by two. Low pass filtered coefficients are stored on the left part of the matrix and high pass filtered on the right. Then it is followed by filtering the subimage along the y-dimension and decimated by two. Finally, the image has been split into four bands denoted by LL, HL, LH, and $\mathrm{HH}$, after one level of decomposition. The LL band is again subject to the same procedure. This process of filtering the image is called pyramidal decomposition of image. This is depicted in Fig. 1. The reconstruction of the image can be carried out by reversing the above procedure and it is repeated until the image is fully reconstructed

\begin{tabular}{|c|c|c|}
\hline LL & LH & \multirow{2}{*}{ LH } \\
HL & HH & \\
\hline \multicolumn{2}{|c|}{$\mathrm{HL}$} & HH \\
\hline
\end{tabular}

Figure 1. Spectral decomposition and ordering

\subsection{Lifting Scheme Haar Transform[13]}

The wavelet lifting scheme is a method for decomposing wavelet transforms into a set of stages. The forward lifting wavelet transforms starts with a split step, which divides the data set into odd and even elements. The predict step uses a function that approximates the data set. The difference between the approximation and the actual data replaces the odd elements of the data set. The even elements are left unchanged and become the input for the next step in the transform. The update step replaces the even elements with an average. This results in a smoother input for the next step of the next step of the wavelet transform. The odd elements also represent an approximation of the original data set, which allows filters to be constructed. The simplest version of a forward wavelet transform expressed in the lifting scheme is shown in figure 2 .

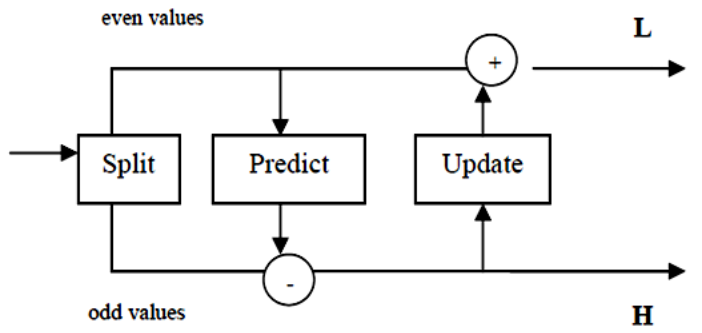

Figure 2. Lifting Scheme Forward Wavelet Transforms
In the lifting scheme version of the Haar transform, the prediction step predicts that the odd element will be equal to the even element. The difference between the predicted value (the even element) and the actual value of the odd element replaces the odd element. For the forward transform iteration $\mathrm{j}$ and element $i$, the new odd element, $j+1, i$ would be

$$
\operatorname{odd}_{\mathrm{j}+1, i}=\operatorname{odd}_{\mathrm{j}, \mathrm{i}}-\operatorname{even}_{\mathrm{j}, \mathrm{i}}
$$

In the lifting scheme version of the Haar transform the update step replaces an even element with the average of the even/odd pair (e.g., the even element si and its odd successor, si+1):

$$
\operatorname{Even}_{j+1, i}=\left(\operatorname{even}_{j, i}+\operatorname{odd}_{j, I}\right) / 2
$$

The original value of the oddj,i element has been replaced by the difference between this element and its even predecessor. Simple algebra lets us recover the original value:

$$
\operatorname{Odd}_{\mathrm{j}, \mathrm{i}}=\operatorname{even}_{\mathrm{j}, \mathrm{i}}+\operatorname{odd}_{\mathrm{j}+1, \mathrm{i}}
$$

Substituting this into the average, we get

$$
\begin{gathered}
\operatorname{Even}_{\mathrm{j}+1, \mathrm{i}}=\left(\operatorname{even}_{\mathrm{j}, \mathrm{i}}+\operatorname{even}_{\mathrm{j}, \mathrm{i}}+\operatorname{odd}_{\mathrm{j}+1, \mathrm{I}}\right) / 2 \\
\text { Even }_{\mathrm{j}+1, \mathrm{I}=}=\operatorname{even}_{\mathrm{j}, \mathrm{I}}+\left(\operatorname{odd}_{\mathrm{j}+1, \mathrm{I}} / 2\right)
\end{gathered}
$$

The averages (even elements) become the input for the next recursive step of the forward transform. This is shown in Figure 3.

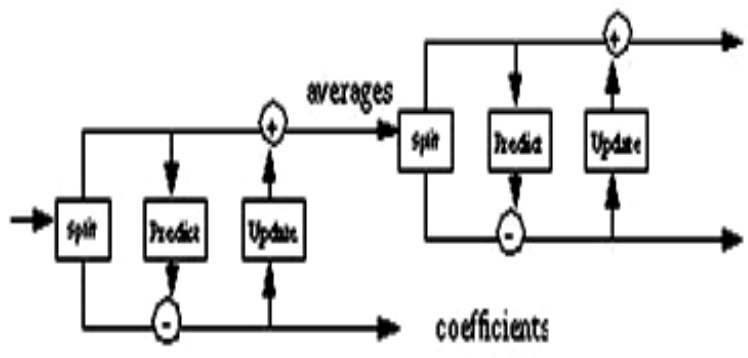

Figure 3. Two steps in wavelet lifting scheme forward transform

One of the elegant features of the lifting scheme is that the inverse transform is a mirror of the forward transform. In the case of the Haar transform, additions are substituted for subtractions and subtractions for additions. The merge step replaces the split step.

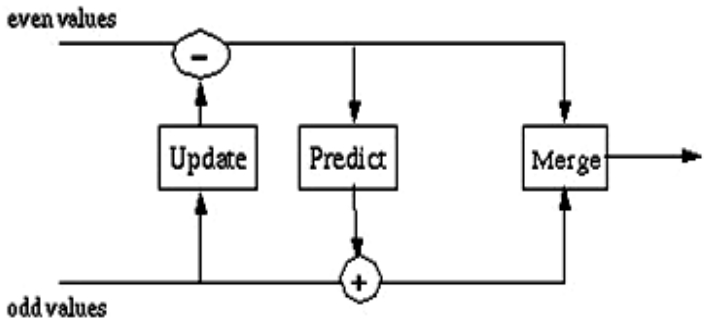

Figure 4: lifting Scheme inverse transform 


\subsection{ASWDR encoding[3][4]}

There are many coding Schemes for wavelet coefficients are available. Image coding is computationally simple and effective technique which utilizing scalar quantization on hierarchical structures of transformed images. Shapiro was the first to introduce such a technique with his EZW algorithm[3]. Different variants of this technique have appeared in the literatures which provide an improvement over the initial work. SPIHT, WDR, ASWDR are some of the examples. Here We have used one of the most recent coding scheme which is ASWDR. The main aim of ASWDR is to improve the subjective perceptual qualities of compressed images and improve the results of objective distortion measures. ASWDR was able to encode more values than WDR. This gives an a posteriori validation of the predictive scheme employed by ASWDR[4]. The creation of the new scanning order only adds a small degree of complexity to the original WDR algorithm. Moreover, ASWDR retains all of the attractive features of WDR: simplicity, progressive transmission capability, and ROI capability. Below I have describe the steps needed in ASWDR algorithm.

Step 1 (Initialize). Choose initial threshold, $\mathrm{T}=\mathrm{T} 0$, such that all transform values satisfy $|\mathrm{w}(\mathrm{m})|<\mathrm{T} 0$ and at least one transform value satisfies $|\mathrm{w}(\mathrm{m})|>=\mathrm{T} 0 / 2$. Set the initial scan order to be the baseline scan order.

Step 2 (Update threshold). Let $\mathrm{Tk}=\mathrm{Tk}-1 / 2$.

Step 3 (Significance pass). Perform the following procedure on the insignificant indices in the scan order:

Initialize step-counter $C=0$, Let Cold $=0$

Do Get next insignificant index $m$ Increment step-counter $C$ by 1 If $|w(m)|>=T k$ then Output sign $w(m)$ and set $w Q(m)=T k$

Move $m$ to end of sequence of significant indices

Let $n=C-$ Cold, Set Cold $=C$

If $n>1$ then Output reduced binary expansion of $n$ Else if

$|w(m)|<T k$ then
Let $w Q(m)$ retain its initial value of 0. Loop until end of insignificant indices

Step 4 (Refinement pass). Scan through significant values found with higher threshold values $\mathrm{Tj}$, for $\mathrm{j}<\mathrm{k}$ (if $\mathrm{k}=1$ skip this step). For each significant value $\mathrm{w}(\mathrm{m})$, do the following:

If $|w(m)| \epsilon[w Q(m) ; w Q(m)+T k)$, then Output bit 0 Else if $|w(m)| \epsilon[w Q(m)+T k ; w Q(m)+2 T k)$, then Output bit 1 Replace value of $w Q(m)$ by $w Q(m)+T k$.

Step 5 (Create new scan order). For each level j in the wavelet transform (except for $\mathrm{j}=1$ ), scan through the significant values using the old scan order. The initial part of the new scan order at level $\mathrm{j}-1$ consists of the indices for insignificant values corresponding to the child indices of these level $\mathrm{j}$ significant values. Then, scan again through the insignificant values at level $\mathrm{j}$ using the old scan order. Append to the initial part of the new scan order at level $\mathrm{j}-1$ the indices for insignificant values corresponding to the child indices of these level $\mathrm{j}$ significant values. Note: No change is made to the scan order at level $\mathrm{L}$, where $\mathrm{L}$ is the number of levels in the wavelet transform.

Step 6 (Loop). Repeat steps 2 through 5.

\section{PROPOSED SUPER RESOLUTION ALGORITHM}

Here I have proposed one interpolation algorithm which work well for natural as well as artificial images. As shown in below figure, we take SR matrix with size $2 \mathrm{~m}-1 \times 2 \mathrm{n}-1$ and fill the pixels according to the equation given below. Instead of finding average value of neighbouring pixels, we can use maximum value or median value of neighbouring pixels and implement the same algorithm. We can use wavelet based Interpolation [6] method also to do super resolution.

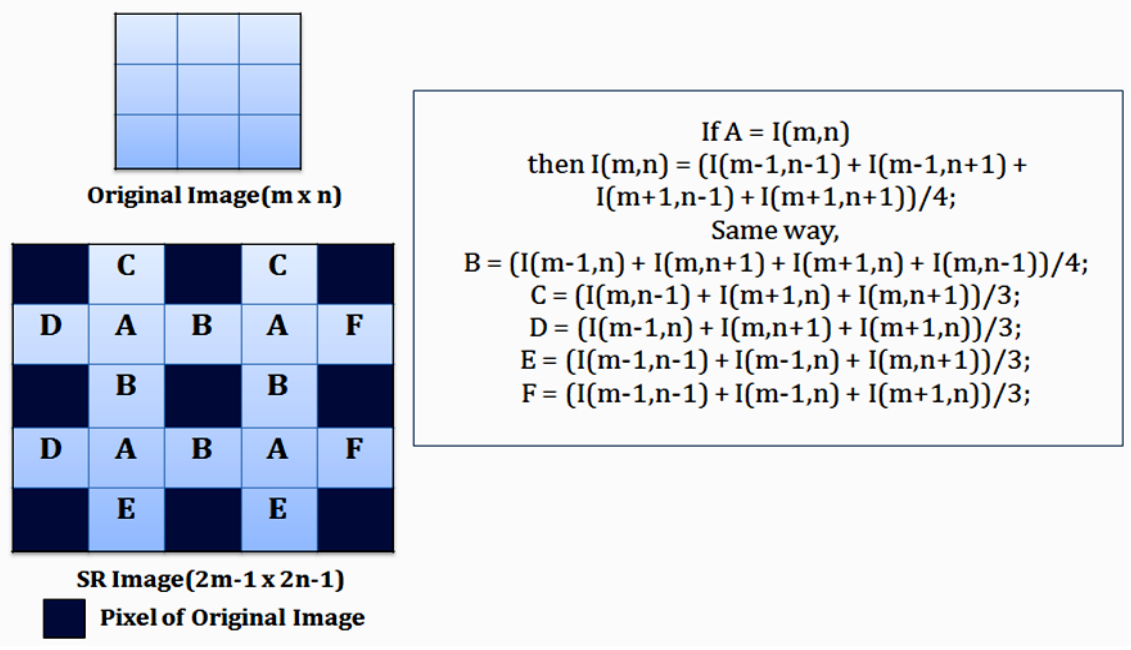

Figure 5. Proposed SR method 
Below we have proposed one algorithm which do super resolution using HAAR wavelet lifting scheme.

Step 1 : Take three input images. Noisy, low resolution blurred, rotated, shifted and compressed images are considered.

Step 2 : First preprocessed input images using i. Apply registration method given in[12] ii. On noise image apply Gaussian filter iii. On blurred image apply IBD algorithm.

Step 3 : Using HAAR wavelet lifting scheme decompose the registered low resolution images.

Step 4 : Encode each low resolution image using ASWDR.

Step 5 : Fused the decomposed images using any of the fusion Rule shown in figure 6 . We have used maximum rule.

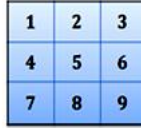

Image I1

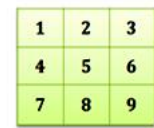

Image 12

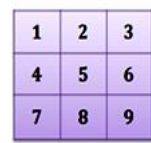

Image I3

Fusion Rules
Average
$\mathrm{I}(\mathrm{i})=(\mathrm{I} 1(\mathrm{i})+\mathrm{I} 2(\mathrm{i})+\mathrm{I} 3(\mathrm{i})) / 3$
Maximum
$\mathrm{I}(\mathrm{i})=\max (\mathrm{I} 1(\mathrm{i}), \mathrm{I} 2(\mathrm{i}), \mathrm{I} 3(\mathrm{i}))$
Median
$\mathrm{I}(\mathrm{i})=\operatorname{median}(\mathrm{I} 1(\mathrm{i}), \mathrm{I} 2(\mathrm{i}), \mathrm{I}(\mathrm{i}))$

Figure 6 . Fusion Rules
Step 6 : Apply inverse lifting scheme to obtain the fused Image. Step 7 : Inverse ASWDR is used to decode fused image.

Step 8 : Apply contrast enhancement technique and perform restoration.

Step 9 : Obtain Super Resolution image by method explained above.

\section{5 . EXPERIMENT RESULTS}

For the experiment purpose we have consider high resolution lena and monalisa images. From this images three noisy, blurred, under sampled, compressed LR images are created of size $256 \times 256$. At the end of algorithm we will get $512 \times 512$ SR image as output. We have tested using both motion blur as well as with Gaussian blur. Gaussian white noise with SNR is added to the blurred low resolution images. We have done experiment on i3 processor with $1 \mathrm{~GB}$ nvidia graphics card. We have chosen PSNR,MSE and ISNR as parameter to evaluate algorithm. The PSNR is defined as:

$$
P S N R=20 \cdot \log _{10}\left(M_{i} X_{i} / \sqrt{ } M S E\right)
$$

The Improvement in Signal-to-Noise Ratio (ISNR) is defined as:

$$
I S N R=10 \log _{10}{ }^{2} /^{2}
$$

Where $\mathrm{j}$ and $\mathrm{i}$ are the total number of pixels in the horizontal and vertical dimensions of the image; $f(i, j), y(i, j)$ and $g(i, j)$ are the original, degraded and the restored image.

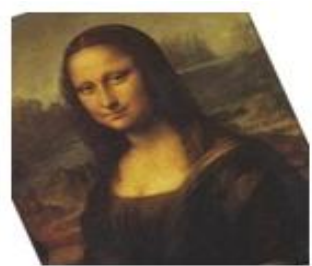

tilt

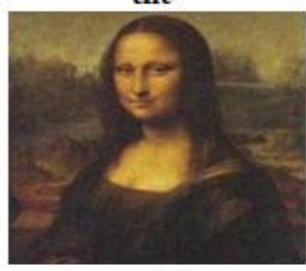

noise

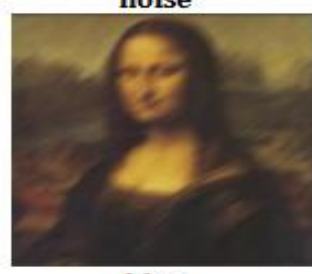

blur

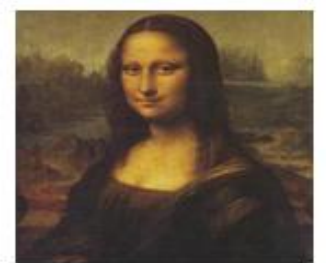

rotated

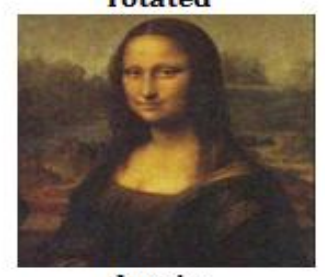

denoise

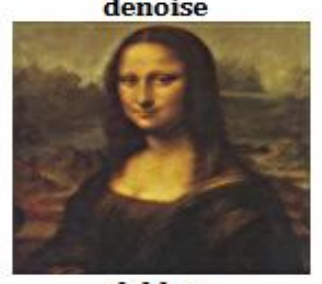

deblur

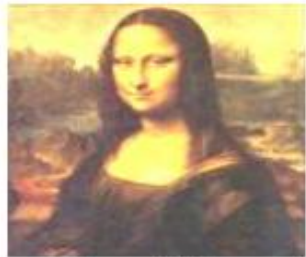

Fused(LL)

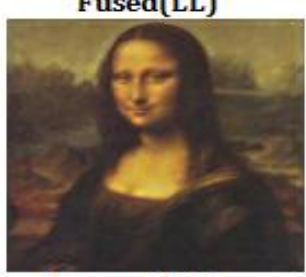

Inverse lifting

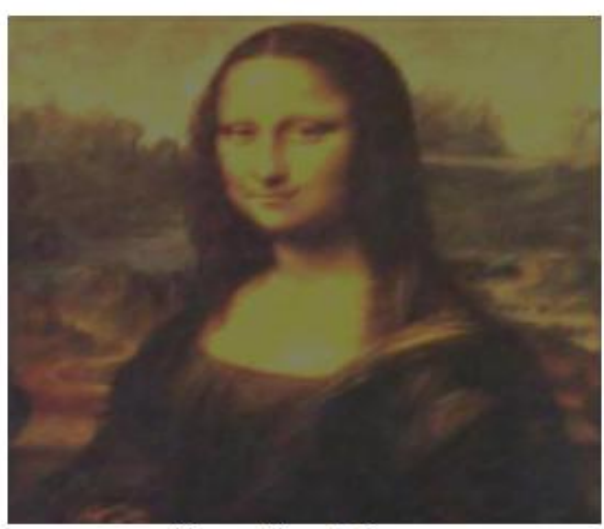

Super Resolution 


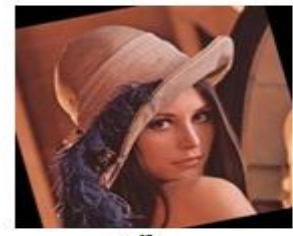

tilt

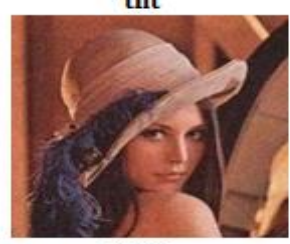

noise

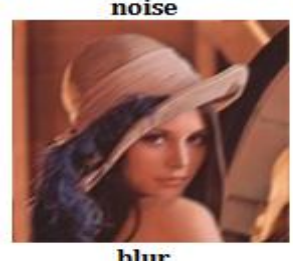

blur

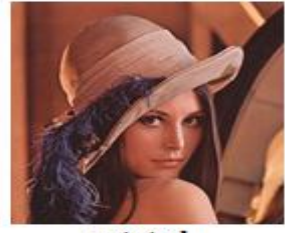

rotated

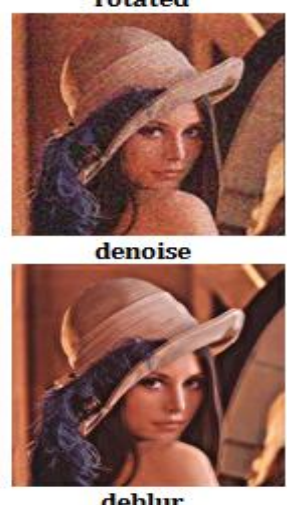

deblur
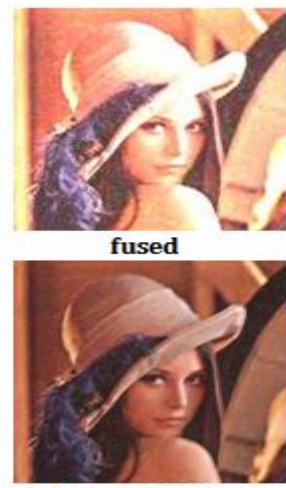

Inverse lifting

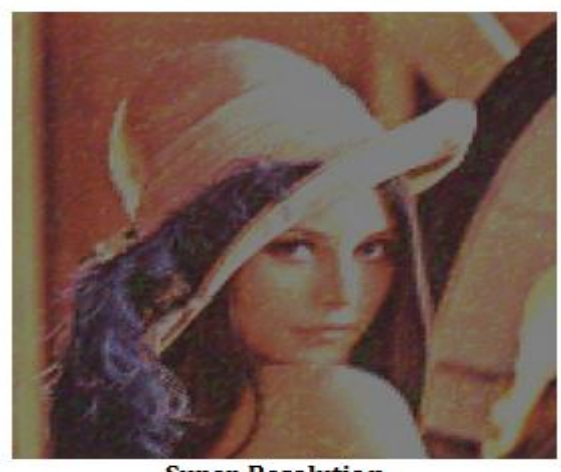

Super Resolution

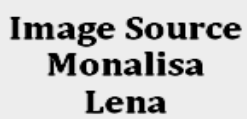

Image Source Lena
MSE

0.8152

0.7072
PSNR(dB)

49.16

45.74
ISNR(dB)

4.41

3.16

Figure 7. Results

\section{CONCLUSION}

The SR image reconstruction is proved to be very useful in many practical cases where multiple frames of the same scene can be obtained. Lifting schemes is used for the reconstruction of a high resolution image and that is the advantage of the proposed method as Wavelet lifting schemes are easier to implement, the inverse transform has exactly the same complexity as the forward transform, requires less memory, faster and can be used on arbitrary geometries. We have done testing on lena image and found that our proposed super resolution reconstruction has given good PSNR. In our future studies we would like to apply other SR methods and wavelet filters for possible image sharpening and enhancement.

\section{REFERENCES}

[1] Rohit Arora, Madan lal Sharma, Nidhika Birla, "An Algorithm for Image Compression Using 2D Wavelet Transform", International Journal of Engineering Science and Technology (IJEST) ISSN : 0975-5462 Vol. 3 No. 4 Apr 2011 pp. 2758 - 2764.

[2] Vinay U. Kale, Nikkoo N. Khalsa, "Performance Evaluation of Various Wavelets for Image Compression of Natural and Artificial Images" ,International Journal of Computer Science \& Communication, Vol. 1, No. 1, January-June 2010, pp. 179-184.

[3] R.Sudhakar, Ms R Karthiga, S.Jayaraman, "Image Compression using Coding of Wavelet Coefficients - A Survey", ICGST-GVIP Journal, Volume (5), Issue (6), June 2005, pp.25-38.

[4] James S. Walker, "Wavelet-based Image Compression", Sub-chapter of CRC Press book: Transforms and Data Compression.
[5] Ashwini M. Deshpande, Suprava Patnaik, Comparative Study and Qualitative-Quantitative Investigations of Several Motion Deblurring Algorithms,2nd International Conference and workshop on Emerging Trends in Technology (ICWET) 2011,(IJCA),pg 27-34.

[6] Ping-Sing Tsai, Tinku Acharya," Image Up-Sampling Using Discrete Wavelet Transform", 9th JCIS 2006, 8 to 11 October 2006.

[7] S. C. Park, M. K. Park, and M. G. Kang, "Super-resolution image reconstruction: A technical review," IEEE Signal Processing Mag., vol. 20, pp. 21-36, May 2003.

[8] Sina Jahanbin, Richard Naething, "Super-resolution Image Reconstuction Performance", May 16, 2005

[9] S. Chaudhuri, Ed., Super-Resolution Imaging. Norwell, MA: Kluwer, 2001.

[10] S.P. Kim, N.K. Bose, and H.M. Valenzuela, "Recursive reconstruction of high resolution image from noisy undersampled multiframes," IEEE Trans.Acoust., Speech, Signal Processing, vol. 38, pp. 1013-1027, June 1990.

[11] Ayers G. R., and Dainty J. C. Iterative blind deconvolution methods and its applications. Optics Letter, vol. 13, no. 7, July 1988.

[12] E.D. Castro, C. Morandi, "Registration of translated and rotated images using finite Fourier transform", IEEE Transactions on Pattern Analysis and Machine Intelligence 700-703,1987.

[13] A.Jensen, A.la Courharbo, “ Ripples in Mathematics : The Discrete Wavelet Transform”, springer,2001. 